\title{
LA SYSTÉMIQUE : UN OUTIL POUR UNE POSTURE ENSEIGNANTE CRITIQUE EN ARCHITECTURE
}

\author{
Author(s) / Auteur(s) : \\ Dorra ISMAIL \\ PhD, Maître de Conférences \\ Directrice du 4C-ENAU \\ Responsable de l'équipe de recherche EaE \\ Co-fondatrice de ebniecolo.tn (1 $1^{\text {re }}$ plateforme sur l'éco-construction en Tunisie) \\ École Nationale d'Architecture et d'Urbanisme de Tunis (ENAU) \\ Université de Carthage (UCAR) \\ ismaildellagidorra@gmail.com
}

\begin{abstract}
Résumé :
Re-penser le monde implique une disposition à adopter une posture critique (Taddei, 2010) d'une manière systémique (globale et située à la fois) en tant qu'individu, citoyen ou professionnel engagé dans une discipline et un savoir-faire/être donné. Dans notre cas, il s'agit du savoir en rapport avec l'architecture, les modes d'établissements humains et leur "enseignabilité" à venir ou à penser.

La posture critique n'est pas un savoir ou un objet disponible, c'est un positionnement, un processus souvent individuel, pouvant devenir collectif. Ce qui est à l'essence d'une posture critique c'est la volonté de dépasser un état de fait, d'innover et de comprendre. En architecture ce dépassement est indispensable pour être à l'écoute de l'évolution de notre métier, du monde qui bouge numériquement et écologiquement.

En Tunisie, La crise du COVID-19 a impulsé un mouvement de solidarité et d'intelligence collective pour pallier à la pénurie du matériel paramédical. Architectes, nous nous sommes retrouvés au centre d'une dynamique nationale de fabrication de visières et de masques par imprimante 3D. Cet élan a dégagé l'expression d'une posture collective.

Le post-covid se mesure, de notre point de vue, dans l'intérêt qu'accordent les tunisiens, les architectes et les étudiants à la question écologique en architecture. Davantage de maître d'ouvrages, ONG, Municipalités, décideurs institutionnels nous sollicitent aujourd'hui et interagissent avec notre plateforme ebniecolo.tn. Alors que cela fait plus de vingt ans que nous tentons d'initier les étudiants et d'inciter les architectes à re-penser nos modes constructifs et architecturaux à fort impact carbone, malgré une faible réceptivité.

Cette "événementialité en acte" (Ismaïl, 2009) post-covid19 nous pousse à adopter une posture critique "systémique" en vue d'une transition écologique dans une pensée collective et coopérative. Nous exposerons le contour épistémique, les outils conceptuels et les paliers significatifs pour la construction d'une posture critique enseignant(e), comme support pour engager un débat.
\end{abstract}

Keywords / Mots-clés :

posture critique, positionnement Post-COVID19, événementialité en acte, pensée collective, transition écologique, architecture

\section{POSTURE CRITIQUE ENSEIGNANT(E) VS APPRENANT(E) : LA SYSTEMIQUE UN OUTIL}

La systémique souvent considérée comme un champ scientifique à part, est traversée par plusieurs courants de pensées, cycles, typologies et niveaux holistiques depuis les premières compréhensions dans les années 1950 avec les systèmes d'analyses de Ludwig von Bertalanffy, Cependant, outre ces étapes constitutives et historiques de la pensée systémique, nous aimerions revenir vers la définition première du terme systémique : une manière d'appréhender les phénomènes dans leur ensemble, soit intégrer les phénomènes dans leurs interactions et selon une approche macro ( $c f$. par exemple Ouali, 2020). 
Cette compréhension première (au sens du verbe) nous interpelle et nous aimerions la convoquer ici afin de traduire une manière d'appréhender par un positionnement, mais également un outil à la disposition de tou.te.s pour penser autrement, c'est-à-dire ensemble, dans une intelligence collective (Staune, 2019) le champ architecture Post-COVID-19.

Pour Daniel Krob (2009), l'approche système est d'abord "un paradigme de pensée, c'est-à-dire une manière d'appréhender de façon homogène une certaine catégorie d'objets 'dynamiques"'. Le paradigme architectural ou du champ "architecture" est, de ce fait, lui aussi à comprendre en tant que paradigme (Pierre Boudon, 1992) de pensée à actualiser avec le monde en devenir (Deleuze, 1969; Morin, 2003) dont le COVID-19 a dessiné certains incompressibles (Ismaïl, 2016).

En effet, peut-on aujourd'hui en tant qu'architectes, enseignant.e.s ou chercheur.e.s, penser notre architecture, le fait constructif, la ville, les connexions socio-urbaines, indépendamment d'une pensée écologique ? La question est comment l'architecte peut-il créer le "cadre" pour une pensée systémique faisant de l'intégration des matériaux Biosourcés et sains, l'aménagement écologique des sites des projets, l'autonomisation énergétique des bâtiments, la récupération des eaux pluviales, le recours aux énergies renouvelables..., comme partie prenante de la production architecturale (projet système).

Krob parle d'architecte système qui s'occupe des interfaces (sous-systèmes d'ingénierie) et leur donne un cadre. Pour Krob (2009, p.14): "Un mauvais cadre ne saurait typiquement que conduire à un "mauvais" système". Le schéma de Krob met en évidence ce "cadre" et les deux systèmes (technique $\&$ humain) en jeu dans un projet système.

Néanmoins, il nous semble que le volet technique et celui humain ne suffisent pas pour consolider le rôle de l'architecte-système dans le projet-système à visée écologique.

Ces deux volets doivent impérativement s'inscrire dans une gouvernance-système (le cadre) pouvant les accompagner et leur faciliter le déploiement et l'intelligibilité.

Dans une autre mesure, nous avions développé dans nos travaux antérieurs sur les événementialités (Ismaïl, 2009), la nécessité d'inscrire la compréhension d'une figure architecturale par rapport aux trajectoires constitutives (politiques, juridiques, sociales, conjoncturelles, techniquestechnologiques...) qui délimitent sa compréhension, et ce, dans la durée (les trajectoires constitutives prennent des valeurs et des épaisseurs différentes selon les temporalités).

Cependant, il y a une trajectoire ou une épaisseur qui devient prégnante par rapport à d'autres et c'est la prégnance de la conscience écologique collective au cours et post crise covid-19 (mars/mai 2020), en tant qu'évémentialité en acte, qui nous intéresse à ce niveau.

L'expérience de l'impression des visières de protection par l'imprimante 3D, impulsée par des architectes en Tunisie et développée sur tout le territoire et dans plusieurs universités tunisiennes, a mis en évidence un cadre particulier: l'architecte joue un rôle prépondérant et dynamiseur dans la "situation" (le projet système à cadrer) sanitaire installée par la pandémie COVID-19 ?

Depuis, deux ou trois années l'usage de l'imprimante 3D est courant dans les recherches en Biotechnologie, Biochimie et en médecine.

Aujourd'hui, les prothèses dentaires, se font par impression numérique.

Lorsque nous avions lancé l'appel sur FB ( $c f$. Fig. 1) pour imprimer les visières et tout matériel médical et paramédical nécessaire (à l'instar de la Corée du Sud, de l'Italie et plus tard d'autres pays comme le Maroc, l'Algérie), il y eut un engouement et un effet d'entraînement très particulier. Plus de 3000 partages et interactions. Plusieurs personnes nous ont contacté d'Egypte, d'Algérie, de la Syrie...

Cet engouement nous a fait prendre conscience que l'intelligence collective se développe lorsque le besoin et les enjeux ${ }^{1}$ sont partagés d'une manière claire et évidente par tou.t.e.s. Dire que c'est facile, partager les fichiers HTML, montrer que cela sert et permet de répondre à une problématique partagée

\footnotetext{
1 Idem en pédagogie. Ainsi, dans l'article "La complexité comme outil pour former des architectes-citoyens autonomes" (Ismail, 2017), nous développons l'importance d'une coopérativité entre enseignant.e.s et apprenant.e.s sur les diagnostics, les objectifs et les méthodes pédagogiques pour une meilleure interaction pédagogique et de meilleurs résultats (mesurables).
} 
par tous, à savoir: se protéger, permet de sensibiliser, de convaincre, de créer un engouement collectif. L'ingénieur Sud-Africain, entrepreneur et chef d'entreprise visionnaire, Elon Musk est un pionnier dans cette posture, dans la mesure où il diffuse et partage tous ses plans et modèles, notamment pour le premier modèle de voiture Tesla3 électrique, en 2018. Cette posture a totalement bouleversé les industries automobiles et les réflexes conventionnels de protection de la propriété intellectuelle et industrielle.

Aussi, le pic d'intérêt donné à nos deux publications sur les réseaux sociaux Facebook en date du 17 \& 22 mars 2020, montre aussi que les solidarités dans le partage de l'information, le savoir et le savoirfaire libèrent les personnes et les jeunes aux talents exceptionnels (les ingénieurs en mécatronique qui ont dessiné les masques et objets paramédicaux sur Solidworks à notre demande, les ingénieurs en génie électronique qui ont breveté la conception d'une imprimante $3 \mathrm{D}$, etc.).

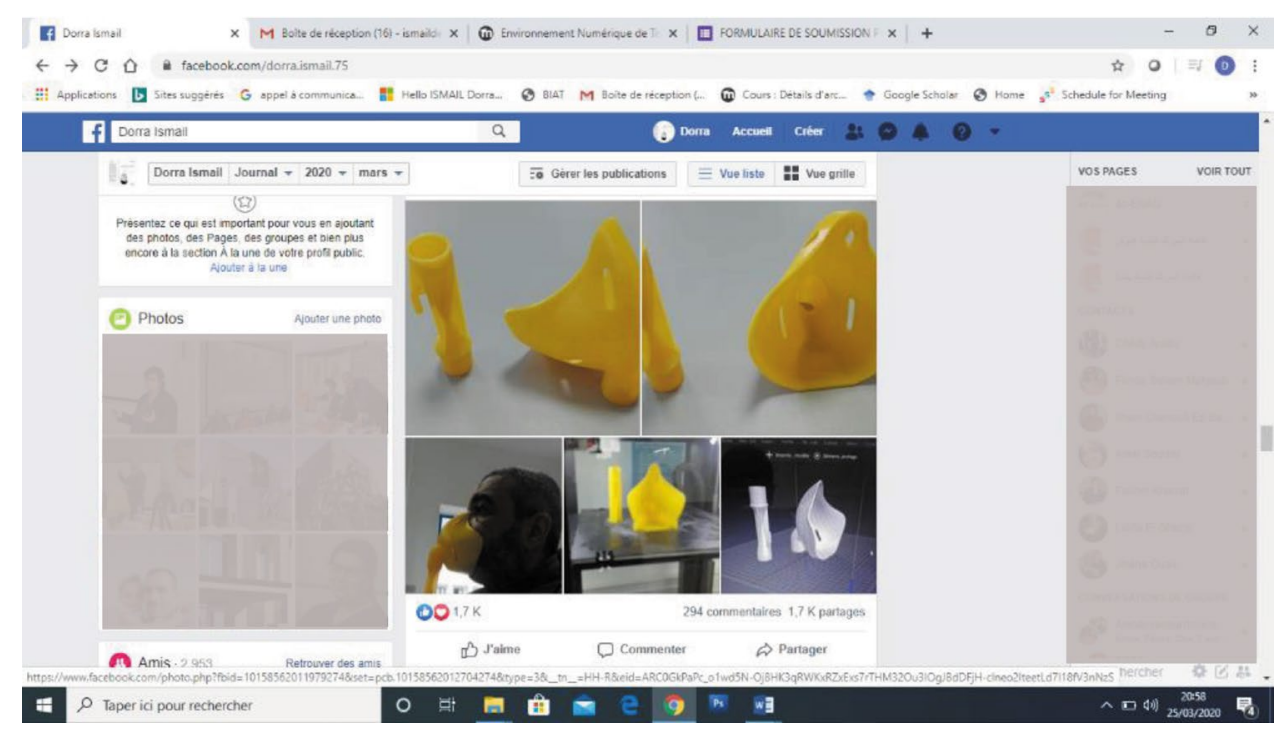

Fig. 1 - Une des publication sur les réseaux sociaux FB lors de la $1^{\text {re }}$ vague de la pandémie.

Différents prototypes ont été réalisés lors de la période de confinement depuis le 17/03/2020, fruit d'un travail de plusieurs jours d'une équipe pluridisciplinaire (architectes, ingénieurs et étudiants en géniemécatronique, IA, médecins, industriels).

Un projet PRF (Projet de Recherches Fédérées) a été déposé au Ministère de la recherche ("Programme R\&I COVID-19") pour valider cette collaboration interdisciplinaire et pour répondre d'une manière durable et systémique aux besoins urgents d'aujourd'hui et de préparer les besoins de demain en termes paramédicaux (tels que le Masque Respiratoire en cours de conception par une équipe de jeunes étudiant.e.s et chercheurs de l'ENICarthage ou le Masque en plexiglas développé avec notre partenaire socioéconomique A. Design Abdennadher : http://www.a-design.tn/).

Les Prototypes ont été validés par des médecins du service des urgences de l'Hôpital Régional de Zaghouan.

Notre projet PRF Bioplastic_3DPrint, soumissionné dans le cadre de l'appel, consiste à développer le matériau BIOPLASTIQUE ${ }^{2}$ ( $50 \%$ plastique recyclé et $50 \%$ fibres végétales) en tant que matériau

\footnotetext{
2 Le produit Bioplastique permet également de produire des matériaux de construction écologiques et durables (projet QAWS pour VRR 2019. Le Bioplastique a également un fort potentiel d'utilisation dans d'autres domaines tels que le transport, l'aéronautique, voire la biotechnologie et l'agriculture puisque la collecte, le recyclage et la valorisation des déchets de fibres végétales permet de développer une niche socio-économique à fort potentiel de développement et dans le respect des $\operatorname{ODD}(3,8,9,11,12 \& 13)$. L'impression 3D de consommables médicaux et paramédicaux a fait l'objet d'études et recherches pluridisciplinaires Internationales en cours de validation. Voir à ce propos les études sur "La reconstruction faciale assistée par imprimante 3D" (Ernoult et al., 2015) ou sur "Les dispositifs médicaux et modèles anatomiques produits par Imprimante 3D" (Pierreville et al., 2018). "L'innovation et l'intérêt de notre projet c'est le
} 
écologique pour l'usage de l'imprimante 3D en vue d'imprimer des consommables médicaux et paramédicaux pour pallier aux pénuries comme celle vécue lors de la première vague de la crise du COVID-19 (mars/mai 2020).

La production du Bioplastique permet de développer une niche industrielle innovante en Tunisie basée sur le Compoundage (de granulés) tunisien et l'injection compression, respectant les ODD et selon un process industriel, préservant nos ressources naturelles, tout en valorisant les déchets des fibres végétales (Halfa, Margine, Cactus, Dérivés du palmier, Noyaux de Dattes, etc.).

Le projet s'inscrit dans la volonté de trouver des solutions stratégiques et durables pour pallier à la crise du COVID-19 en produisant par Impression 3D des consommables paramédicaux à base de filament en Bioplastique à la place du PLA (et/ou PVA).
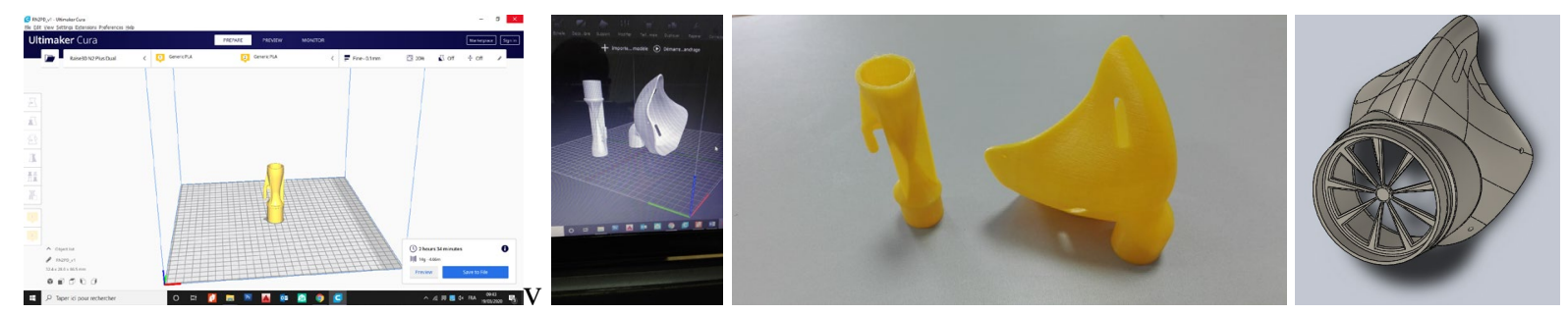

Fig. 2 - Valve de venturi 1 imprimée en 3D - Dessin de Lassad AKERMI (Génie Mécatronique) à partir du fichier OpenSource trouvé par l'équipe. 2. Valve de Venturi + Casque imprimés en 3D - Dessin de Lassad AKERMI (Génie Mécatronique) à partir du fichier OpenSource trouvé par l'équipe. 3. Valve de Venturi + Casque imprimés en 3D. 4. Masque N95 Modélisé par Lassad AKERMI (ENICarthage).
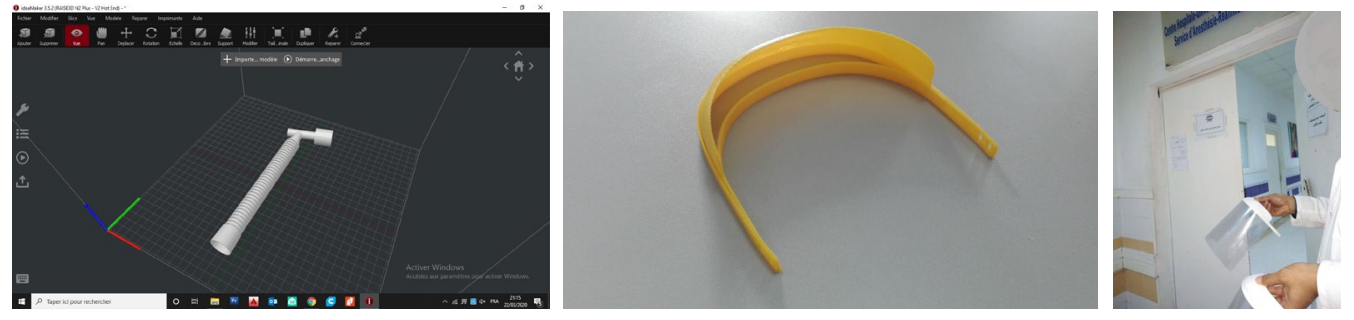

Fig. 3 - 1. Le filtre annelé utilisé dans les hôpitaux, modélisé en autocad par Mehdi Dellagi et retravaillé par Meriem Hedhli de l'ENICarthage sur Solidworks et Catia et imprimée en 3D. 2. Visière pour masque de protection. 3. Masque à visière de protection Imprimé en 3D à partir d'un fichier OpenSource modifié et validé par trois corps médicaux (hôpital Mongi Slim La Marsa, Hôpital Régional de Zaghouan \& Samu de Tunis). 30 Masques imprimés et distribués au 26/03/2020.
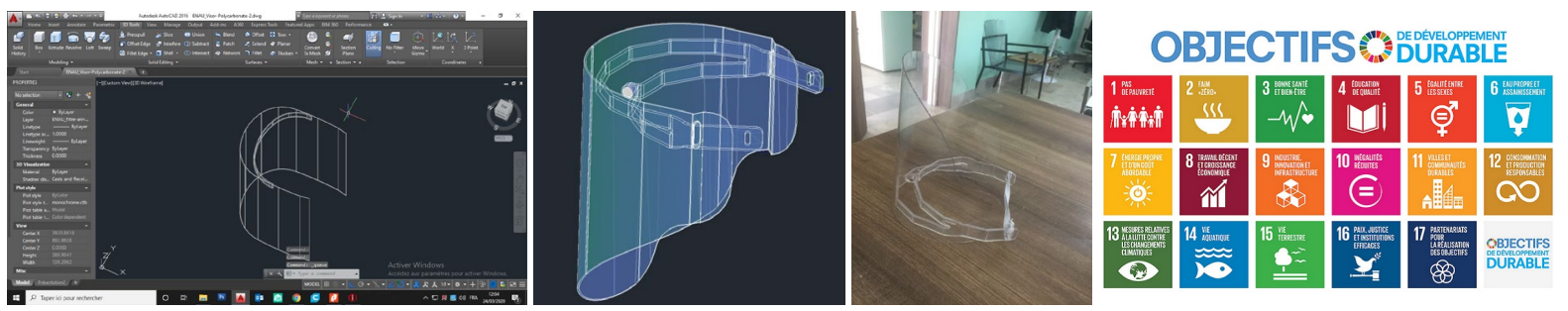

Fig. 4 - 1. Modélisation sur Autocad par Mehdi Dellagi (qartbunDESIGN) d'un moule (pour injection plastique) pour l'industriel Abdennadher. 2-3. Masque en plexiglas $4 \mathrm{~mm} /$ Visière 1,5mm produit par A.Design Abdennadher (partenariat

ENAU/ Habib ABDENNADHER-SA / qartbunDESIGN) pour une production de masques en série (100 en cours).

4. Objectifs de DD. Crédit-photo : internet.

double output : 1. La validation d'un produit écologique Le Bioplastique à fort potentiel de développement industriel ; 2. Tout en apportant un perfectionnement et une généralisation de l'utilisation de l'imprimante 3D, pour des produits médicaux et paramédicaux de première urgence, et optimisés par l'industrie locale dans ces temps de crise. D'où l'intitulé Bioplastic_3DPrint". Extrait du projet PRF déposé (Chef de Projet : Dorra Ismaïl). 
Notre projet PRF "Bioplastic 3DPrint" n'a pas été retenu (Argument : il manque un laboratoire de recherche par rapport au quota demandé).

Le projet a donc été rejeté, essentiellement pour une question organisationnelle/administrative davantage que pour des raisons scientifiques (pourtant le ministère de tutelle est bien au fait que l'ENAU unique école d'architecture publique du pays, ne dispose pas encore de Laboratoire de recherche en fonctionnement et que la discipline architecture (création du doctorat en 1995) est en cours de constitution).

De notre point de vue, nous estimons qu'il est inapproprié de juger ce projet comme tout autre projet présenté par une équipe médicale ou paramédicale.

Les enjeux systémiques ne sont pas les mêmes.

Cela dénote aussi que le paradigme de pensée systémique doit être intégré aux différents niveaux organisationnels, institutionnels, opérationnels et donc de gouvernance (échelle Macro-systémique).

Si les institutions, elles-mêmes peinent à voir l'intérêt d'un projet système par rapport à une vision globale de l'instant c'est que l'exceptionnalité du moment n'est pas saisie (l'événement qui crée l'occasion et inversement).

Ce qui peut être retenu de cette expérience : une pensée système ou une approche système doit être elle-même intégrée à l'échelle macro-économique et politique et impacter la gouvernance (gouvernance-système), sans quoi, elle reste un sous-système n'ayant pas d'impact majeur, dans la durée, ni sur la société ni dans une intelligence collective.

Léon Laulusa (2020) parle de nouveau modèle de société post-covid et de homo oeconomicus: "Dans ce contexte de grandes mutations, un consensus semble émerger en faveur d'un nouveau modèle de société homo reconomicus, différent du capitalisme actuel, comme illustré par ce titre du Financial Times du 18 septembre 2019 sur la réinitialisation du capitalisme : 'Capitalism : time for a reset'".

Pour nous cet homo oeconomicus sera essentiellement écologique.

Cette crise/pandémie va nous rassembler dans une conscience collective si chère à Edgar Morin lorsqu'il évoquait dans les années 2000 la nécessité d'éduquer pour l'ère planétaire par rapport à la question écologique (Diamer, 2020) et le destin planétaire de tous les humains.

Néanmoins, afin que les sociétés s'inscrivent dans ce "destin" planétaire homo oeconomicus, il faut d'abord qu'il soit intelligible au niveau de la Société-Etat et Citoyen-Institution-Etat.

Si la discipline architecture n'est pas intelligibilisée à l'échelle nationale et institutionnelle comme une "architecture système" (KROB, 2009), la question durable et écologiste restera juste une dynamique ponctuelle et selon les niveaux d'interactions entre les systèmes, mais pas systémique, pour impacter l'ensemble de la société et enclencher la transition écologique.

Le moment du COVID-19 ( $1^{\text {re }}$ vague) était une occasion pour enclencher cette approche systémique, mais malheureusement les éléments du système architecture n'étaient pas suffisamment élaborés pour impulser cette pensée systémique globale. Le bâtiment est un enjeu-climat majeur dans ce système global puisqu'à l'échelle mondiale, les bâtiments consomment $32 \%$ de l'énergie globale finale et $25 \%$ d'émission de gaz à effet de serre (Lucon, 2014).

La pandémie va-t-elle déplacer les réflexes et inscrire un nouveau paradigme de pensée systémique de la fabrique du bâtiment et de nos villes ?

Cet épisode nous révèle que ce déplacement n'est, malheureusement, pas pour tout de suite. 
Néanmoins, pour l'appli ebniecolo.tn (https://ebniecolo.tn/) on peut dire qu'il y a un avant et un après COVID-19 (plus exactement après le moment : épisode des visières par imprimante 3D) mais, l'impulsion systémique globale (échelle macro ${ }^{3}$ ) n'est pas encore manifeste.

En effet, de plus en plus de personnes, institutions, $\mathrm{ONG}^{4}$, médias ${ }^{5}$, nous contactent et sollicitent nos conseils sur l'éco-construction.

\section{LE CONTOUR EPISTEMIQUE D'UNE POSTURE CRITIQUE INCONTOURNABLE}

Dans le contexte, incertain, mouvant, complexe qui est le nôtre, comment se prémunir, et résister à la consommation non filtrée des fake news et des infinités d'informations Big Data qui envahissent notre quotidien et notre cerveau?

Il y a urgence à centrer l'apprentissage et les Skills des enseignants et à fortiori, des apprenant.e.s, sur comment adopter une posture critique (dans notre article de 2017 nous avions parlé de comment former des architectes autonomes et des paliers significatifs à adopter en situation d'enseignement) et une capacité à prendre une distanciation pour séparer, nuancer, re-composer et re-construire par nos propres moyens les données à traiter.

Pour cela, il y a trois niveaux de compétences à considérer :

- le niveau systémique macro-disciplinaire ${ }^{6}$ en architecture : un cadre (système d'enseignement) apte à développer et intégrer des multi-compétences réflexives et critiques, pouvant synthétiser les acquis et connaissances transversales de différentes disciplines (Sciences des systèmes \& pensée complexe, Sciences de l'éducation, Avancées en neurosciences, Neuro-phénoménologie, Biomimétisme, directives et conclusions de $\mathrm{l}^{1} \mathrm{OCDE}^{7} \&$ du GIEC, Gouvernance éducative mondiale et tunisienne, posture écologique (constructive), Ethique ${ }^{8}$ et technologie) au service d'un projet commun, d'un besoin et/ou objectif collectif ${ }^{9}$.

- le niveau systémique micro-disciplinaire en architecture : compétences à s'adapter aux nouveaux enjeux et situations en vue d'intégrer des savoirs et savoir-faire en ingénierie

3 Outre le fait qu'il y ait, récemment (Septembre 2020), création d'un comité de réflexion (dont nous sommes membre) au sein de la DGBC (Direction Générale des Bâtiments Civils, sous l'impulsion de Madame Sondès Béji Kraiem, Directeur au sein de la DGBC et partenaire du 4C-ENAU (dont nous assurons la Direction depuis Avril 2019) sur l'intégration de matériaux alternatifs dans les cahiers de charges réglementant les marchés publics.

4 Projet éco-construction et Permaculture à Zaghouan.

5 Un Journaliste Mohamed. B.K. nous a contacté au mois de septembre 2020 pour nous demander conseil en marge de sa préparation d'une émission sur l'éco-construction (12 à 24 séquences) sur la chaine de télévision nationale visant à diffuser et vulgariser les modes constructifs alternatifs pour des logements soutenables et économiques. Cette volonté est un indicateur sur un besoin qui se fait de plus en plus ressentir quant à la nécessité de construire et habiter "autrement" aujourd'hui en Tunisie, car le bâtiment est le $1^{\text {er }}$ consommateur énergétique (en Tunisie) et touche en premier lieu le citoyen dans la pyramide de Maslow.

6 Jihène Ouali (2020) démontre d'une manière scientifique cette évidence/vérité "déclarative", en se basant sur la modélisation systémique de Jean-Louis Le Moigne et de Courtès, sur l'impact de l'objet-système architecture à l'échelle macro sur l'ensemble des sous-systèmes qu'ils soient d'ordre organisationnels, socio-économiques ou disciplinaires.

7 Les études et données de l'OCDE tendent vers une conscientisation des milieux éducatifs et scientifiques de la nécessité d'un nouveau paradigme pédagogique et de pratiques enseignantes. Les sciences de l'éducation, les études en neurosciences et en neuro-phénoménologie donnent un cadre scientifique, éthique et réflexif pour cette nouvelle posture enseignante (Taddei, Meirieu). En effet, les avancées scientifiques récentes en biomécanique ou en épigénétique, redéfinissent le débat éthique et philosophique humaniste tout en donnant à cette époque post-pandémie des arguments scientifiques pour repenser notre modèle de société local et global à la fois.

8 https://usbeketrica.com/fr/article/tout-ingenieur-devra-demain-integrer-les-enjeux-climatiques-et-energetiques-a-sonmetier consulté le 2020-09-20.

9 Dans le cadre du 4C-ENAU (https://www.4c.tn/) nous tentons de donner forme à cet objectif par des formations certifiantes et des événements (chantiers-école, encadrement, initiation à l'entrepreneuriat et accompagnement des étudiants et des enseignants à la mise en place de projets R\&D et de Valorisation des Résultats de Recherche, etc.) dans le cadre de l'éco-innovation en architecture. 
alternatives alliant Big-data, Ios, TIC, Industrie 4.0 et techniques énergétiques pour les villes de demain durables, résilientes et connectées.

- le niveau systémique du leadership: compétence à former des apprenant.e.s autonomes, aptes à réfléchir par eux-mêmes que ce soit à l'intérieur d'un système disciplinaire ou à l'extérieur de lui (niveau savoir-être de Bloom).

\section{LES OUTILS CONCEPTUELS \& LA PENSÉE SYSTÈME OU COMMENT AMORCER LE CHANGEMENT DE POSTURE CRITIQUE ?}

Pour mener l'apprenant.e. vers une attitude réflexive et des compétences "augmentées" (Laulusa, 2020), il est indispensable de l'impliquer dans le processus de réflexion avec ses différents niveaux (objectifs, contenus, méthodes et outils utilisés) ; A. Diemer parle de "System Thinking".

L'EAD (enseignement à distance) est une occasion qui nous incite à re-penser la scénarisation de nos cours et leurs paliers significatifs.

Le support pédagogique devient à la fois un moyen, une occasion et un prétexte ${ }^{10}$ (Ismaïl, 2016) selon des variables différentes pour enclencher une réflexion sur un savoir (savoir-faire) donné.

Aussi, pour le cours de troisième année l'objectif global ${ }^{11}$ étant au niveau macro-disciplinaire, dans la mesure où nous expliquons aux apprenants que le cours est un prétexte, mais également un moyen ${ }^{12}$ pour les sensibiliser à la posture écologique.

D'emblée nous les impliquons dans le processus et la finalité visés par le cours : le positionnement écologique.

Les étapes sont des paliers, soit des sous-systèmes du système architecture dans lequel nous cherchons à les impliquer.

Chaque sous-système devient un point d'entrée (par induction: du particulier au général ou par déduction : du général au particulier) pour mesurer leurs aptitudes à intégrer certaines connaissances et compétences complexes.

Dans ce qui suit, nous allons développer les trois paliers adoptés dans le cadre du cours de détail(s) pour les étudiant.e.s de troisième année architecture.

\section{Palier 1 : déclencher le processus de posture critique}

Lors de la première séance, nous entamons un débat sous forme de brainstorming "apprentissageaction sur le détail de la Gerberette ${ }^{13}$ de Beaubourg pour inciter l'apprenant.e. à aborder les œuvres à partir d'un spectre plus élargi que celui de la fonction, de la forme ou de l'image "superficielle", en intégrant la notion de contexte (conjoncture socio-politique, concours, lieu, topo-morphologie, coordination architecte-ingénieur, singularité des acteurs en jeu,...) et du détail technico-architectural pouvant définir le projet.

En effet, lors du débat en évoquant le projet du centre culturel Beaubourg à Paris nous leur demandons ceci : quelles sont les principales idées qui vous viennent à l'esprit pour décrire cette œuvre ? Les

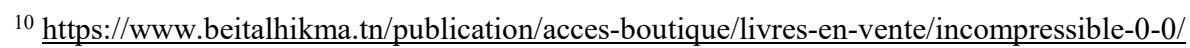

${ }^{11}$ Ce cours cherche à développer, chez l'étudiant.e., la capacité à :

- réfléchir l'aspect constructif dans la démarche de conception : la tectonique étant une notion centrale dans cette initiation ;

- porter une attention particulière sur les matériaux (leur comportement mécanique, énergétique et esthétique), l'ingénierie énergétique (impact carbone, cycle de vie, etc.), thermique et de confort d'une conception ;

- maitriser la combinaison de trois niveaux : le souci du matériau, la compréhension du système constructif et leur rapport au potentiel d'expression architecturale, en vue d'une nouvelle approche du détail d'architecture par le point d'entrée de la pensée écologique.

${ }^{12}$ Avec des occasions/situations : les exercices, d'applications, les chantiers-école, les références, les différentes ressources, etc.

${ }^{13}$ Un ouvrage Incompressible 0.1 sur l'enseignement du cours de détail( $(s)$ est en phase de recherche d'éditeur.
} 
réponses sont de différents ordres: "La structure apparente", "La circulation extérieure", "Les plateformes", "La placette"...

Des réponses vagues et non structurées selon un cheminement analytique, voire "systémique", qu'il soit lié à l'histoire de l'œuvre, le contexte du concours, le positionnement et le référentiel des architectes, les problèmes résolus par le projet ou les problèmes engendrés par le projet, etc...Les réponses restent donc à un niveau superficiel, voire micro, c'est soit l'aspect fonctionnel, soit celui formel (sémiotique) qui est mis en avant.

Ces mêmes réponses, constituent un point d'entrée pour évoquer la nécessité d'appréhender les choses d'une manière plus globale (complexe) en situant un "artéfact architectural" dans son contexte d'apparition et en différenciant les différents niveaux de compréhension (Ismaïl, 2009).

Aussi, nous évoquons à ce sujet :

- dans un premier temps : le vide qui a été possible grâce à un système constructif et spatial qui a pu dégager une partie du terrain (malgré un programme chargé) : d'où le niveau territorial, urbain et topographique dans la compréhension de l'originalité de la réponse et de son intérêt par rapport à la situation ;

- dans un deuxième temps : le détail de "Gerberette" (la quasi-totalité des étudiant(e)s ignorent ce détail technique et à la fois conceptuel qui a permis la réalisation des plateformes libres de points d'appuis intermédiaires et constitue un élément essentiel dans le process conceptuel).

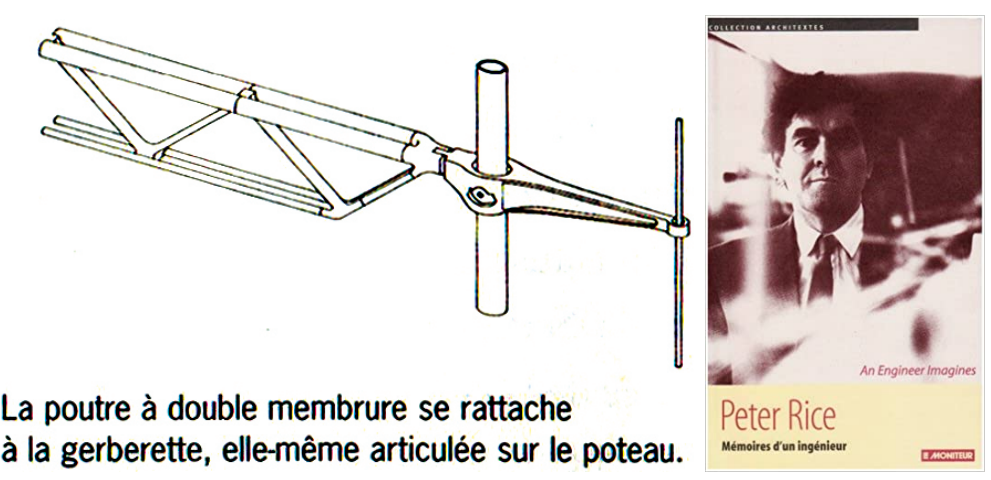

Fig. 5 - La Gerberette : pièce-clé du projet.p.24. In : RICE.S. Peter Rice. Mémoires d'un ingénieur, (1998 pour l'édition en langue française), éditions Le Moniteur, Paris. b-.Couverture du livre Peter Rice Mémoires d'un ingénieur. Peter Rice a joué un rôle de premier plan dans le processus du projet et de mise en œuvre de la Gerberette. Néanmoins, les étudiant.e.s en architecture à l'ENAU ne connaissent pas Peter Rice ni son rôle dans le projet.

Cet apprentissage-action permet d'installer l'apprenant.e. dans une posture d'interrogation et de recul par rapport à ses mécanismes habituels de réflexion et de compréhension.

Attirer l'attention de l'apprenant.e, le bousculer, fait partie des buts intermédiaires pour installer une coopérativité enseignant-apprenant selon un positionnement visé : le point de vue tectonique et écologique dans l'appréhension de l'architecture.

Ce but étant atteint, il est possible après cela de passer à d'autres paliers ${ }^{14}$ en vérifiant si certaines notions sont assimilées.

\footnotetext{
${ }^{14} \mathrm{~L}$ 'article "La réflexivité et la complexité pour former des architectes citoyens autonomes" (Ismaïl, 2017) a permis de délimiter les étapes pour échafauder les paliers significatifs pour distancier l'apprenant(e) d'une posture systématique visant à répéter les réflexes de conception par "extrude".
} 


\section{Palier 2 : compréhension par induction (du détail vers le principe) ; Niveau Savoir (Taxonomie de Bloom)}

Être en mesure de différencier entre l'enveloppe, le système constructif et le couvrement d'une architecture.

Ce palier passe par un exercice d'application afin de valider et re-construire sur la base des écarts identifiés.

Les écarts permettent d'identifier les difficultés des étudiant.e.s (au niveau du dessin, de la compréhension, de la distinction entre l'enveloppe et le couvrement ou l'enveloppe et le système constructif). Le but ultime étant de donner à l'apprenant.e. les moyens pour nuancer par lui-même et dans toute situation conceptuelle ou réflexive ces niveaux.

Les réponses des étudiants sont un point de départ (moyen) pour aborder les notions relatives aux systèmes constructifs en nous basant sur leurs propres erreurs/écarts (technique du Learning by doing) et développer leur savoir (compréhension).
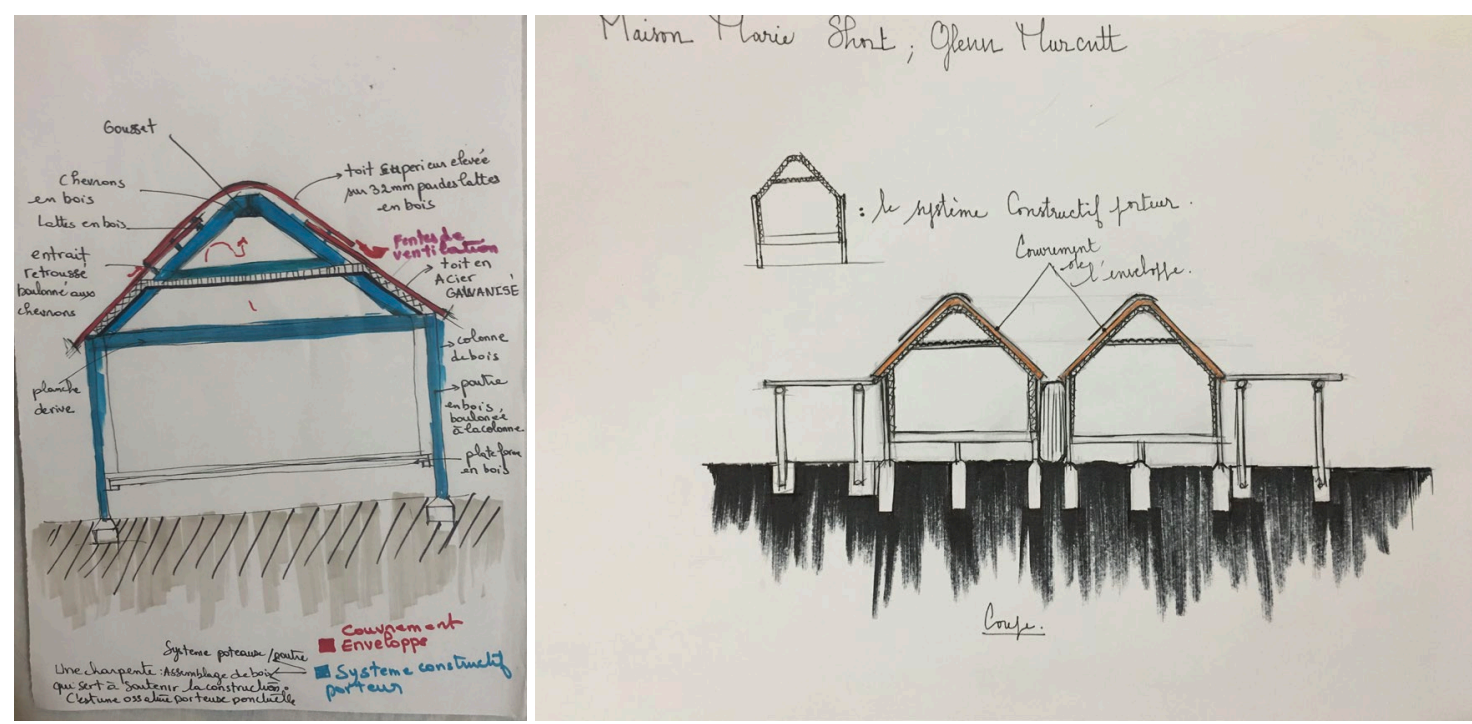

Fig. 6 - Dessins d'étudiants de troisième année. AU. 2020/21. Catégorie de réponse 1 : certains étudiant.e.s reproduisent les dessins sans répondre à l'objectif de l'exercice et ne différencient pas l'enveloppe, le couvrement et le système constructif. Ils confondent l'ossature porteuse en bois de la structure secondaire en bois pour poser le couvrement voûté.

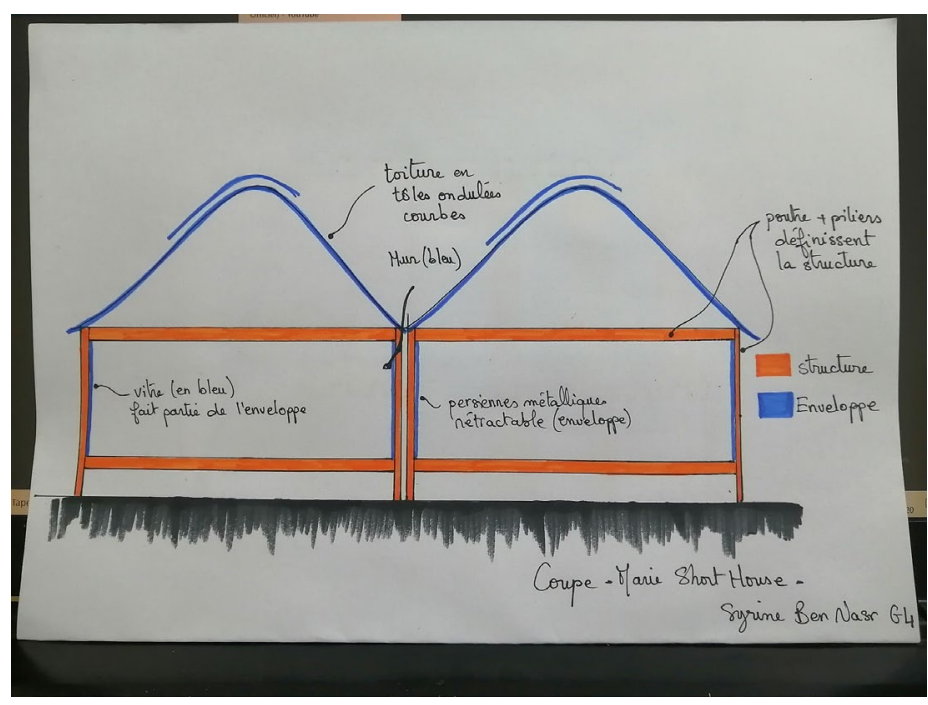

Fig. 7 - Dessin d'étudiante de troisième année. AU. 2020/21. Catégorie 2 : d'autres étudiant.e.s. redessinent la coupe au premier niveau (formel) sans nuancer les niveaux. 
Palier 3 : savoir-faire par extrapolation ;

Niveau savoir-faire (Taxonomie de Bloom)

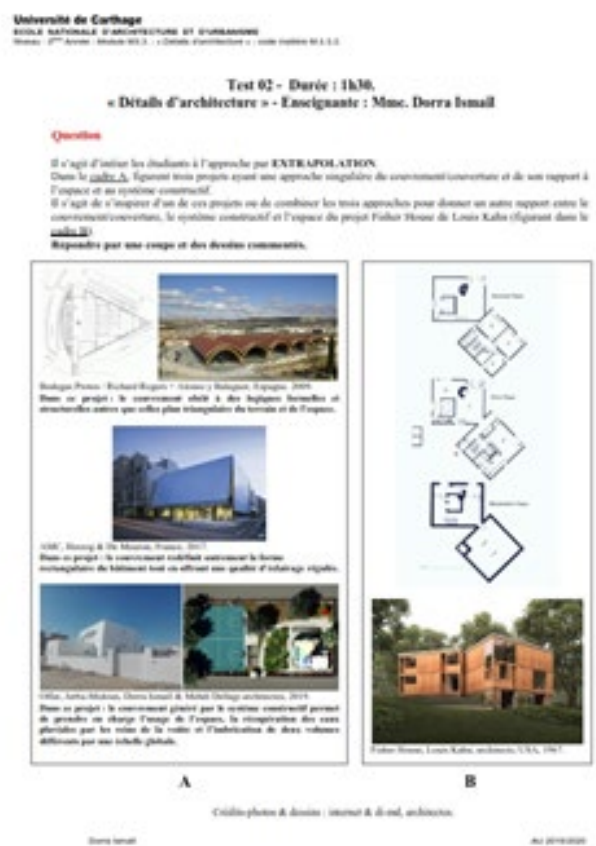

Fig. 8 - Enoncé de l'exercice dont l'objectif et de les initier à la notion d'extrapolation en leur demandant de proposer une interprétation du couvrement du projet de Louis Kahn en intégrant la notion de bioclimatique. Des projets de références (tunisiens et étrangers) donnent des repères du point de vue de l'approche bioclimatique, du rapport couvrement/espace, etc.

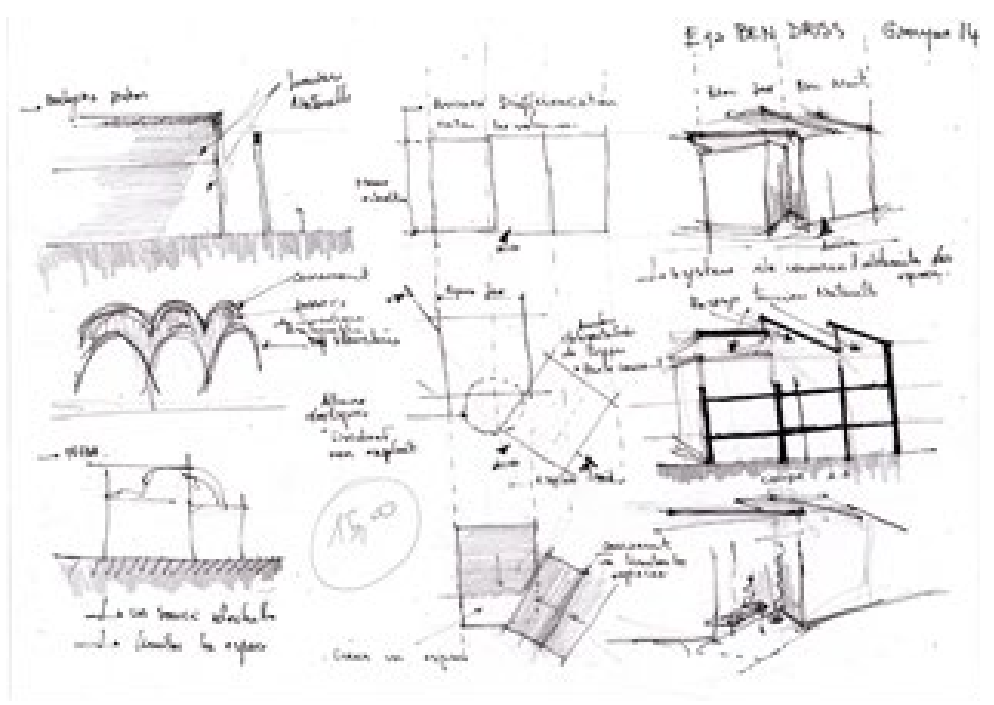

Fig. 9 - Travaux d'étudiants de $3^{\text {e }}$ année architecture 2019/2020. Les étudiant.e.s expérimentent la notion d'extrapolation tout en focalisant le savoir/compréhension d'une œuvre sur des dimensions étudiées précédemment (matériau, ventilation, couvrement, système constructif, enveloppe, etc.) autres que formalistes. Ils passent donc du niveau de savoir vers le savoir-faire. 


\section{Palier 4 : l'extrapolation comme outil pour le savoir-être ; Niveau savoir-être (Taxonomie de Bloom)}

Cette phase est celle de "l'auto-régulation" et on observe que l'étudiant.e. lors de cet exercice (examen final) maîtrise à la fois le dessin, les principes bioclimatiques et l'outil "extrapolation".

La démarche par paliers permet de scénariser l'objectif pédagogique du cours dans son ensemble et dans ses étapes.

Cette scénarisation est systémique et réflexive, dans la mesure où elle combine des niveaux de compréhension et d'apprentissages complexes dans un but bien précis.

Ces outils réflexifs ont été développés grâce à nos travaux de recherche sur "l'événementialité" en architecture (Ismaïl, 2009).

En effet, positionner une œuvre architecturale (considérée ou élevée au statut d'événement architectural) dans le temps et dans l'espace indépendamment des récits tant historiques que littéraires qui l'ont traversée, permet de l'observer avec une distanciation critique.

C'est l'objet de notre recherche (Ismaïl, 2004-2009) qui a permis de porter un nouveau regard sur certaines figures historiques telles que le Dôme de Florence, le Mausolée Oljeitu de l'Azerbidjan Iranien, l'opéra de Sydney ou le Cristal Palace en les inscrivant dans un continuum systémique et non plus linéaire.

Le continuum obéit à des considérations d'autant plus complexes et imbriquées qu'il ne permet plus d'identifier ces figures comme des événements "absolus" (identifiés d'une manière unique et préalablement définie), mais plutôt comme des "événementialités" architecturales au sens où leur lecture est relative selon les catégories qui les traversent d'une manière systémique : événementialité tectonique, événementialité exemplum, événementialité mémorium, etc.

Cette re-considération de l'événement comme objet de réflexion et non plus, objet de consommation uniquement, a donné un cadre systémique en "situation d'enseignement" pour scénariser les objectifs et les contenus pédagogiques en vue d'adopter une posture critique constamment renouvelée.
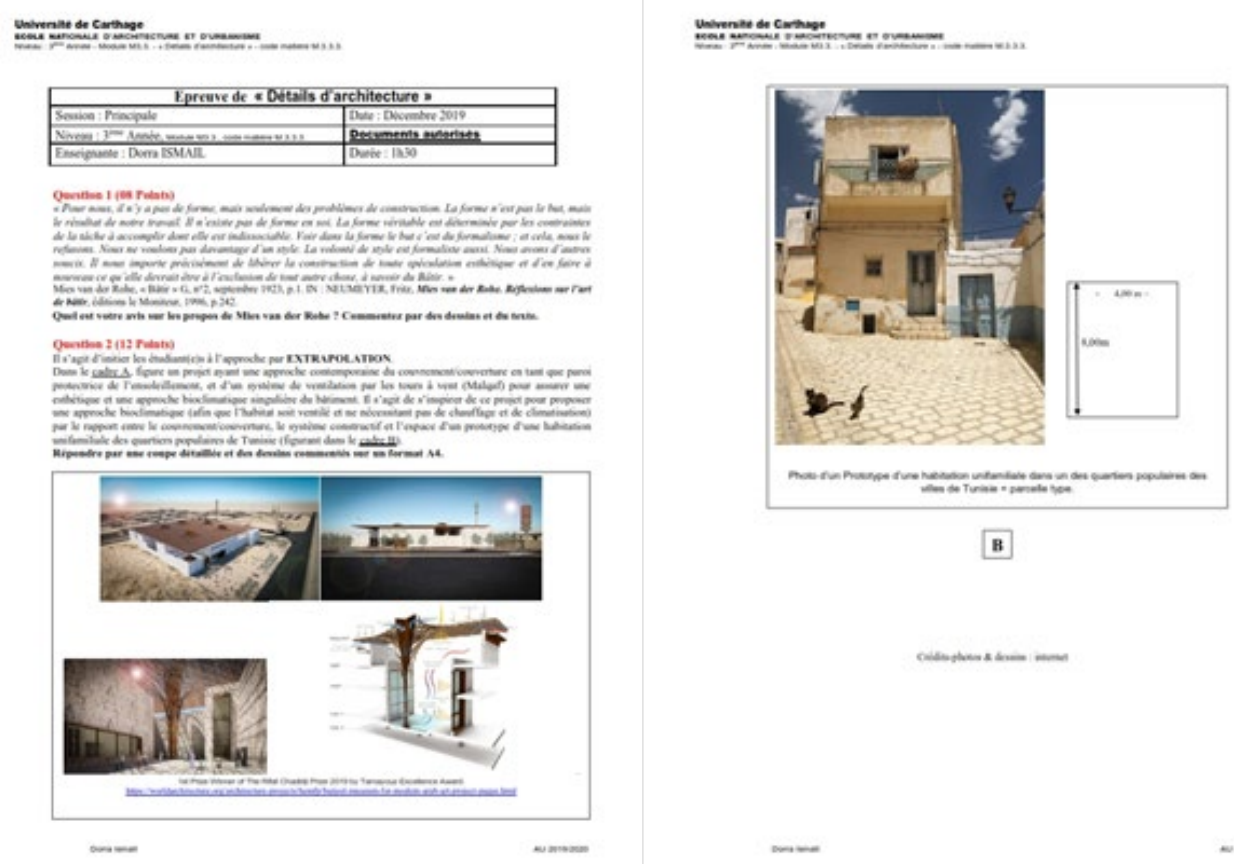

B

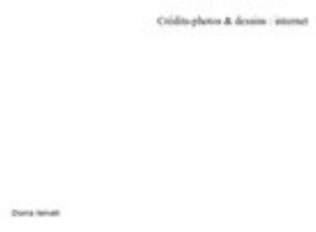

Fig. 10 - Enoncé de l'exercice. Objectif : mettre les étudiant.e.s dans une situation réelle dans le contexte architectural et urbain tunisien où il s'agit d'extrapoler un bâtiment existant en lui donnant une lecture bioclimatique. Un projet de référence est également donné en tant que repère pour l'extrapolation. 


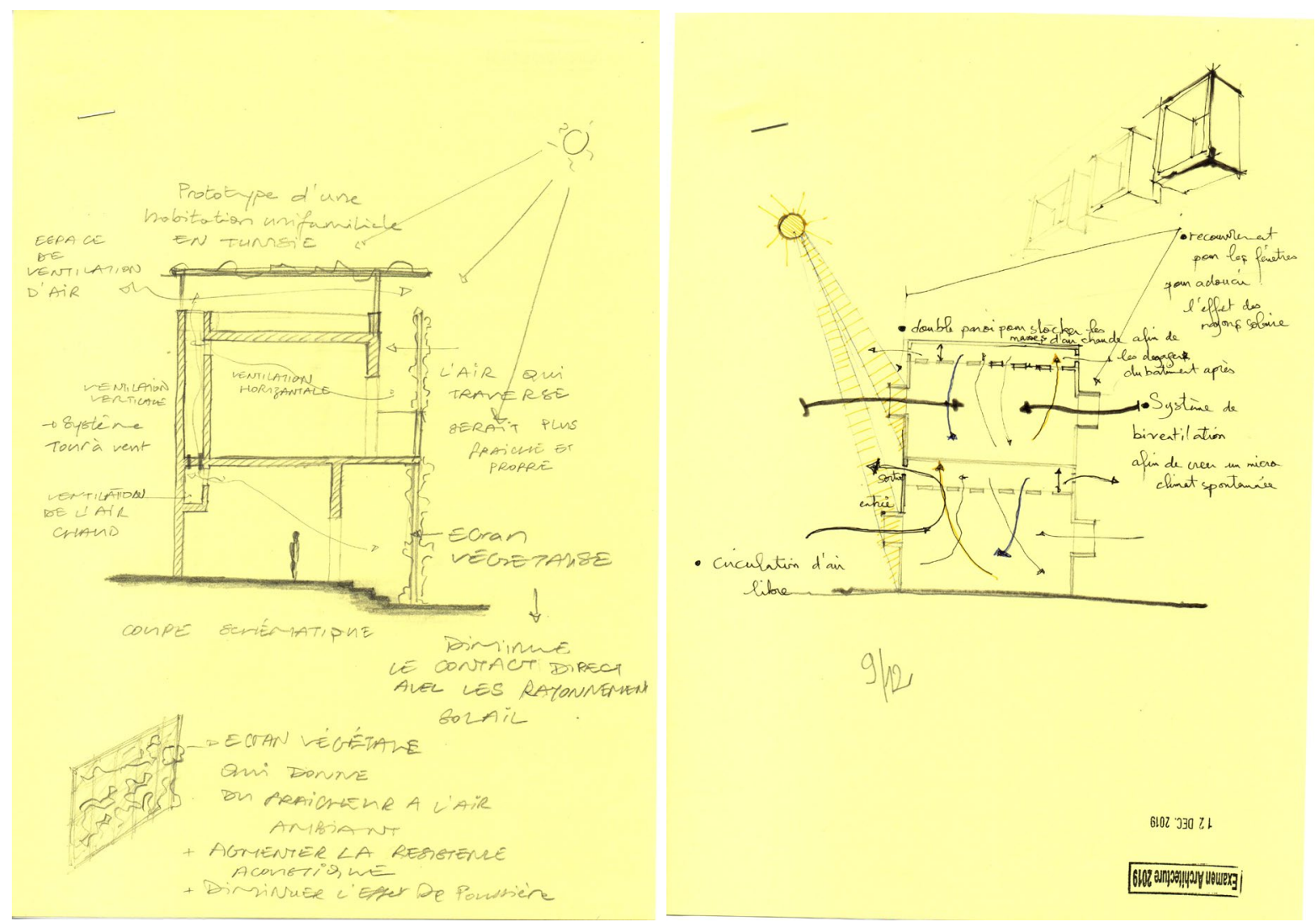

Fig. 11 - Travaux d'étudiants de $3^{\mathrm{e}}$ année architecture 2019/2020. Les étudiant.e.s maîtrisent la notion d'extrapolation tout en développant leur savoir-être : comment ils agiraient en situation, afin de penser et concevoir selon une démarche écoresponsable avec comme outils la différentiation entre système constructif, l'enveloppe et le couvrement pour trouver des solutions innovantes pur rendre le bâtiment bioclimatique.

\section{CONCLUSION}

La pensée systémique est aujourd'hui plus que jamais indispensable pour aider tous les corps de métiers et notamment les architectes et les professionnels de l'enseignement en architecture à adopter une posture créative, ouverte et intégrante des enjeux du moment et futurs.

Pour adopter une "posture critique" systémique, un paradigme système (pensée système) doit pouvoir être appréhendable à différents niveaux de la gouvernance (Macro) vers les sous-systèmes disciplinaires (Micro. L'exercice pédagogique étant un élément de ce sous-système).

Or cette posture système dépend en grande partie d'une volonté et d'un "choix progressiste innovant" souvent incertain (Morin évoque la nécessité d'intégrer l'incertitude comme composante de notre vie et de nos choix) d'accepter et d'intégrer le changement.

Ce choix est également une question de mentalité et de prédisposition à se renouveler.

Notre conviction est que des enseignant.e.s critiques, progressistes avec une pensée multi-compétences continuellement renouvelée (donc augmentée) sont à même de former des architectes d'un demain incertain, mais hautement systémique et créatif par ses connections et ses savoirs Big Data. 


\section{REFERENCES}

BOUDON, P. (1992). Le paradigme de l'architecture. Paris : Balzac.

DELEUZE, G. (1969). Logique du sens. Paris : Minuit (coll. Critique).

DELEUZE, G. (1988). Le Pli, Leibniz et le Baroque. Paris : Minuit (coll. Critique).

DIAMER, A. (2020). "Modéliser le Covid-19. Défis et perspectives", Revue Francophone du Développement Durable, $\mathrm{n}^{\circ}$ 15, Mars 2020, France. p.1-72.

ERNOUlt, C., BOUletreau, P., MEYER, C., AUBRY, S., BRETON, P., BACHElet J.-T. (2015). "Reconstruction assistée par l'impression 3D en chirurgie maxillofaciale". Revue de Stomatologie, de Chirurgie Maxillo-faciale et de Chirurgie Orale, Volume 116, Issue 2, pp.95-102.

ISMAIL, D. (2016). Incompressible 0.0 : réflexions sur l'enseignement en architecture, éditions Beital-Hikma (Académie Tunisienne des Lettres des Sciences et des Arts de Tunis), Tunis.

ISMAIL, D. (2017). "La réflexivité et la complexité pour former des architectes citoyens autonomes".

ISMAIL, D. (2009). La pensée en architecture au 'risque' de l'événementialité. Paris : L'Harmattan.

KROB, D. (2009). "Eléments d'architecture des systèmes complexes". CNRS \& Ecole Polytechnique, France, pp.1-20. http://www.afscet.asso.fr/msc/textes-2009/Krob-elements-archisys.pdf Consulté le 2020-09-20.

LAULUSA, L. (2020). "Managing a post-covid era". ESCP Impact Papers, p.458-464.

MORIN, E. (2015). "Face aux périls 'totaux', nous devons changer de pensée". L'Humanité, France. https://www.humanite.fr/edgar-morin-face-aux-perils-totaux-nous-devons-changer-de-pensee-586523

MORIN, E., MOTTAR, CIURANA, E.T. (2003). Eduquer pour l'ère Planétaire. La pensée complexe comme Méthode d'apprentissage dans l'erreur et l'incertitude humaines, éditions Balland, France.

OUALI, J. (2020). Système de la production architecturale dans le contexte macroscopique: Modélisation et validation dans les discours d'architectes. Thèse de doctorat de l'École Nationale d'Architecture et d'Urbanisme (ENAU), Université de Carthage (UCAR), Tunisie, soutenue le 28/11/2020. [Promotrice : D. Ismaïl Dellagi]

PALlASMAA, J., MALlGRAVE, H.F., ARBIB, M. (2015-2016). Architecture and Neuroscience, edited by Philipp Tidwell, published by the Tapio Wirkkala-Rut Bryk Foundation, TWRB Design Reader, Finland.

STAUNE, J. (2019). L'Intelligence Collective. Clé du monde de demain. Changeons le travail pour changer la société, éditions de l'Observatoire/Humensis, Paris.

LUCON, O. (2014). "Climate Change 2014: Mitigation of Climate Change". Contribution of Working Group III to the Fifth Assessment Report of the Intergovernmental Panel on Climate Change, Cambridge University Press, Cambridge, United Kingdom and New York, NY, USA.

PIERREVILle, J., SERRANO, C., VAN DEN BRINK, H., PROGNON, P., PINEAU, J., MARTELLI, N. (2018). "Dispositifs médicaux et modèles anatomiques produits par impression 3D : quelle diffusion et quelles utilisations dans les établissements de santé français ?". Annales Pharmaceutiques Françaises, Volume 76, Issue 2, pp.139-146.

TADDEI. F. (2020). "Et si l'on considérait les jeunes comme les citoyens de l'éducation?", https://www.innovation-pedagogique.fr/auteur1338.html.

SCHACHTER, V. \& TADDEI, F. (2010). "La créativité dans la recherche", Journal de l'école de Paris du management, 2010/3 (N83), p.29-36. 
